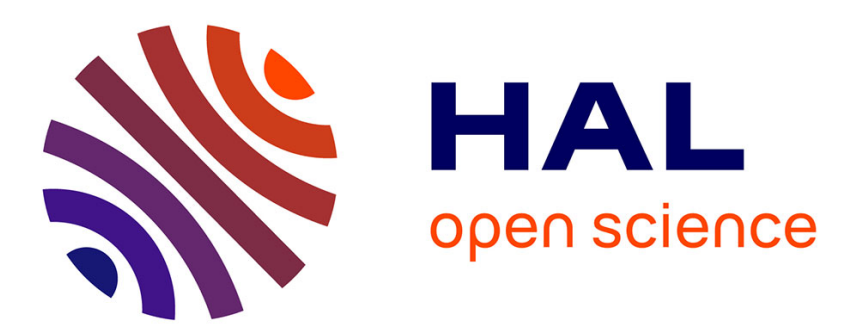

\title{
Routing in a queueing system with two heterogeneous servers in speed and in quality of resolution
}

Benjamin Legros, Oualid Jouini

\section{To cite this version:}

Benjamin Legros, Oualid Jouini. Routing in a queueing system with two heterogeneous servers in speed and in quality of resolution. Stochastic Models, 2017, 33 (3), pp.392 - 410. 10.1080/15326349.2017.1303615 . hal-01728723

\section{HAL Id: hal-01728723 \\ https://hal.science/hal-01728723}

Submitted on 3 Oct 2020

HAL is a multi-disciplinary open access archive for the deposit and dissemination of scientific research documents, whether they are published or not. The documents may come from teaching and research institutions in France or abroad, or from public or private research centers.
L'archive ouverte pluridisciplinaire HAL, est destinée au dépôt et à la diffusion de documents scientifiques de niveau recherche, publiés ou non, émanant des établissements d'enseignement et de recherche français ou étrangers, des laboratoires publics ou privés. 


\title{
Routing in a queueing system with two heterogeneous servers in speed and in quality of resolution
}

\author{
Benjamin Legros $^{a} \bullet$ Oualid Jouini ${ }^{b}$ \\ ${ }^{a}$ PSB Paris School of Business, Department of Economics, 59 rue Nationale, 75013 Paris, France \\ ${ }^{b}$ CentraleSupélec, Université Paris-Saclay, Laboratoire Genie Industriel, Grande Voie des Vignes, \\ 92290 Chatenay-Malabry, France \\ benjamin.legros@centraliens.net• oualid.jouini@centralesupelec.fr
}

March 3, 2017

\begin{abstract}
Heterogeneous servers, in manufacturing and service systems, may have different speeds and different quality levels for the provided service or good. For a two-server queueing model, we formulate the job routing problem for minimizing the stationary weighted sum of the expected time spent in the system and the number of unsatisfied customers per time unit. Using a Markov decision process approach, we prove that the optimal routing policy of jobs to service is a threshold policy that depends on the queue length. When the number of waiting jobs in the queue is below a certain threshold, only one server should work and the other one remains idle. At or above this threshold, both servers should serve jobs. This is an extension of the known result where only the heterogeneity in speed is considered.
\end{abstract}

Keywords: Optimal routing; queueing systems; Markov decision process; threshold policy; heterogeneous servers; quality of resolution.

\section{Introduction}

In manufacturing and service systems, the operation speed usually interacts with the quality of the provided service or treatment. In some cases, high speed may mean not enough attention, which leads to a poor quality of resolution. In other cases, high speed may be related to a well trained and experienced human capacity, which implies high quality of resolution. Examples with speed-quality interaction include call centers where the call conversation duration is correlated to the call resolution probability (de Véricourt and Zhou, 2005; Zhan and Ward, 2014), or healthcare systems where the treatment length may interact with the health deterioration level after the treatment (Yankovic and Green, 2011). 
We consider the problem of dynamically and optimally controlling a queueing system with two heterogeneous servers. Heterogeneity is in terms of speed and quality of the provided service. Managers are then concerned at the same time by the customer sojourn time and the quality of the provided service. In what follows, we review the literature related to this paper.

The slow server problem. This work is most closely related to the slow server problem literature, which has a long history. The slow server problem focuses on routing customers to two heterogeneous servers so as to minimize the mean time in the system. Heterogeneity is in terms of speed, i.e., one server is fast and the other is slow in the sense of mean service time. The main question is what to do with the slow server. For instance, consider the situation where the fast server is busy and the queue is not empty. On the one hand, it is interesting to use the slow server in order to reduce the waiting time in the queue of a given waiting customer. On the other hand, it may also happen that the fast server becomes free after a short time and could have finished the service of this waiting customer before its termination at the slow server.

Krishnamoorthi (1963) was the first to consider the slow server problem. Under elementary assumptions, he shows that the fast server should always be used, and the slow server should only be used when the fast server is busy and the number of customers waiting in the queue exceeds a certain threshold. Rigorous proofs for the optimality of this threshold policy are provided using Markov decision process (MDP) approaches or sample path arguments (Larsen and Agrawala, 1983; Lin and Kumar, 1984; Walrand, 1984). Using value iteration, Koole (1995) provides a simpler version of this proof. Viniotis and Ephremides (1988) consider various extensions of the result, for example, for the case of Erlang servers. Rykov and Efrosinin (2009) also extend the proof of the optimality of a threshold policy including service costs.

Results concerning the optimal routing policy for more than two servers are much more challenging to obtain. The growing dimensionality of the underlying state space is the reason for the difficulty. Weber (1993) uses coupling arguments to show that whenever a job is routed, it should always be routed to the fastest available server; but he only provides a conjecture that the optimal routing follows a state-dependent threshold policy. Two papers claim to have proved the optimality of the state-dependent threshold policy. The first one, Rykov (2001), uses value iteration to show that the optimal value function satisfies monotonicity properties. The second one, Luh and Viniotis (2002), uses a linear programming formulation and sample path analysis. However, de Véricourt and Zhou (2006) prove the incompleteness of the proofs provided in these two papers, and the problem remains open.

Other papers consider the search for good routing heuristics. For the two-server case, Rubinovitch (1985) compares between different non-idling policies so as to determine whether or not the slow server should be used in order to minimize the stationary expected sojourn time in the system. He shows that 
a good policy is a threshold policy based on the traffic intensity. Cabral (2005) extends this result to the multi-server case with uninformed customers. The problem under the Halfin-Whitt heavy-traffic limit regime is also investigated. For a call center application, Armony and Ward (2010) consider a queueing systems with heterogeneous agent pools. They address the customer routing problem subject to a fairness constraint on the workload division. They show that the optimal policy is a threshold policy based on the number of customers in the system. Further asymptotic results include Armony (2005); Atar (2008); Atar and Shwartz (2008).

Recently, the extension of the slow server problem to queueing systems with unreliable servers is addressed. Özkan and Kharoufeh (2014) differentiate the two servers by their service rates and reliability attributes. The slow server is perfectly reliable while the fast server is subject to random failures. The objective is to minimize the stationary average number of customers in the system. Using a Markov decision process approach, the authors prove that it is always optimal to route customers to the fast server when it is available, irrespective of its failure and repair rates. For the slow server, the optimal policy is a threshold policy that depends on the queue length. Other related references include Efrosinin (2013); Özkan and Kharoufeh (2015).

The slow server problem with quality of resolution. Despite its prevalence in practice, the literature has rarely addressed the slow server routing problem by including service quality related factors. Two exceptions, belonging to the call center operations management literature, are de Véricourt and Zhou (2005), and Zhan and Ward (2014). de Véricourt and Zhou (2005) analyze the routing problem in a call center where a customer immediately calls back when her problem is not appropriately resolved. The call quality is defined through a call resolution probability, i.e., the probability that the customer is satisfied and does not call back for the same problem. Servers have different call resolution probabilities and different service rates. They address the dynamic control problem under the objective of minimizing the expected total time of call resolution. For the two-server case, they prove that a threshold policy is optimal. A call should be routed to the server with the highest resolution rate (resolution probability times service rate) whenever possible. The other server will be used only when the number of waiting calls in the queue exceeds a certain threshold. Partial characterization of the optimal policy and practical heuristics are given for the multi-server case.

The resolution rate policy is however shown to perform poorly under an objective that involves the callback probability (Mehrotra et al., 2012). Under the asymptotic many-server quality and efficiency driven regime, Zhan and Ward (2014) extend the analysis of de Véricourt and Zhou (2005), by considering similar modeling and assumptions, but a more general objective in terms of a weighted sum of the expected waiting time and the callback rate. They approximate this asymptotic problem by a diffusion control problem. The efficiency of the analytic diffusion solution is then validated through simulation. 
Although callbacks may be an appropriate measure of quality in some contexts such as technical call centers (Jouini et al., 2008), it is not the case for many other manufacturing and service systems where unsatisfied customers defect rather than comeback to the system. In contrast to the existing literature, in the current paper we assume that a customer does not return after an unsuccessful service. Such situations occur for example in commercial call centers where agents have various selling abilities, or in make-to-order manufacturing firms where an unsatisfied customer with a long lead time may switch to competitors (Hall and Porteus, 2000).

Contributions. Using an MDP approach, we address the optimal routing of customers in service for the two-server problem under the objective of minimizing the stationary weighted sum of the expected time spent in the system and the unsatisfied customer rate. We prove that the optimal routing policy is a threshold policy that depends on the queue length. When the number of waiting customers in the queue is below a certain threshold, only one server should work and the other one remains idle. At or above this threshold, both servers should serve jobs. This is similar to the known result where only the heterogeneity in speed is considered.

The complexity added by the quality of resolution is that one server is not necessarily better than the other one. The value of our analysis in comparison with existing ones is that no assumptions are made on the preference for one given server. The approach for the proof of the threshold policy consists of three steps. In the first step, we prove that the number of busy servers increases with the queue size. In the second step, we prove that under the infinite horizon, having the two servers idling is not optimal. Finally in the third step, we prove that there cannot be changes in the prioritization of one server as a function of the system states.

Structure of the paper. In Section 2, we provide the problem formulation. Using an MDP approach, we prove in Section 3 that the optimal policy is of threshold type. A numerical illustration of the optimal policy is also provided. We finally give a brief conclusion.

\section{Problem Formulation}

Consider a queueing system with a single customer type and two parallel servers, servers 1 and 2 . Customers arrive, at a single first come first served (FCFS) infinite queue, according to a Poisson process with rate $\lambda$. Service times are independent and exponentially distributed with rate $\mu_{i}$ for server $i, i \in\{1,2\}$. Once server $i$ completes a service, the customer is either satisfied with probability $1-\alpha_{i}$, or unsatisfied with probability $\alpha_{i}, i \in\{1,2\}$. An unsatisfied customer defects, and this is considered as a loss of goodwill. To ensure stability, we assume that $\lambda<\mu_{1}+\mu_{2}$. The stationary performance measures of 
interest are the customer expected time spent in the system, denoted by $E(R)$, and the production rate (throughput) of server $i$, denoted by $T_{i}, i \in\{1,2\}$.

Consider now the set of all non-preemptive non-anticipating FCFS routing policies for the routing of customers in service. At any point of time, we want to decide for the first customer in the queue (if any) whether to keep her in the queue, or to serve her by an available server (if any). We combine two objectives to to account for the trade-off between minimizing the time spent in the system and maximizing customer satisfaction about the provided service. Concretely, the goal is to find the optimal routing policy which minimizes the following weighted sum

$$
\alpha_{1} T_{1}+\alpha_{2} T_{2}+c_{R} E(R)
$$

where the coefficient $c_{R}\left(c_{R} \geq 0\right)$ translates the relative importance given, by the system manager, to the expected time spent in the system compared to the number of unsatisfied customers per time unit. Without loss of generality, the cost per unsatisfied customer is one.

We propose to formulate the routing problem as an MDP and next use the value iteration technique to prove the form of the optimal policy. We formulate the problem via the definition of states, the transition structure and the possible actions. In order to completely separate transitions and actions, we allow for idling possibilities, i.e., after an arrival or a service completion there is no automatic routing in service.

States definition. Let us denote by $x$ the number of customers in the queue, $x \geq 0$. The state of the server pair is described through the symbols $0,1,2$ and $1 / 2$. State 0 is a situation where the two servers are idle. State $i$ is a situation where only server $i$ is working, $i \in\{1,2\}$. State $1 / 2$ corresponds to a situation where the two servers are working. A state of the system is thus completely defined by the couple $(i, x), i \in\{0,1,2,1 / 2\}$ and $x \geq 0$.

Transitions. We denote the transition rate from state $(i, x)$ to state $\left(i^{\prime}, x^{\prime}\right)$ by $q_{(i, x),\left(i^{\prime}, x^{\prime}\right)}$. Hence for $i, i^{\prime} \in\{0,1,2,1 / 2\}$ and $x, x^{\prime} \geq 0$, we have

$$
q_{(i, x),\left(i^{\prime}, x^{\prime}\right)}=\left\{\begin{array}{l}
\lambda, \text { if } i^{\prime}=i, x^{\prime}=x+1, \text { for } i \in\{0,1,2,1 / 2\}, x \geq 0, \\
\mu_{1}, \text { if } i=1, i^{\prime}=0 \text { or } i=1 / 2, i^{\prime}=2 \text { and } x^{\prime}=x, \text { for } x \geq 0, \\
\mu_{2}, \text { if } i=2, i^{\prime}=0 \text { or } i=1 / 2, i^{\prime}=1 \text { and } x^{\prime}=x, \text { for } x \geq 0, \\
0, \text { otherwise, }
\end{array}\right.
$$

which corresponds to arrivals and service departures. 
Actions. At each instant of time when at least one customer is in the queue and one server is idling, we are allowed to serve or not this customer and eventually to decide which server to choose. A cost of one is counted per unsuccessful service. This may discourage to route a customer automatically to the first available server. On the other hand, waiting customers incur costs. It is therefore important not to postpone too much a start of service. Hence, decisions have to be taken in situations where (i) at least one customer is in the queue and (ii) at least one server is idle. We have to decide:

- How many customers should be routed in service $(0,1$ or 2$) ?$

- In the case where only one customer should be served, which server should be preferred?

We choose to discretize our continuous-time model. This is possible because it is uniformizable (Section 11.5.2. in Puterman (2005)). We next show that our model satisfies the uniformization condition. In all states where the two servers are busy, there are three possible events: an arrival or a service completion from server 1 or from server 2 . The rate out of each of these states is $\lambda+\mu_{1}+\mu_{2}$. Yet, in all states where only one server is busy, there are only two possible events: an arrival or a service completion from the busy server. When the two servers are idling only an arrival can occur. By adding fictitious transitions from a state to itself we allow that the rate out of each state is $\lambda+\mu_{1}+\mu_{2}$, without exception, for every policy.

We are considering infinite horizon average costs. It is then optimal to schedule customers only at service completion and arrival times: We consider the embedded discrete-time Markov decision chain by looking at the system only at transition instants. They occur according to a Poisson process with rate $\lambda+\mu_{1}+\mu_{2}$. The instantaneous holding costs for the embedded chain count for the whole period until the next transition. If it is optimal to keep a server idle at a given time, then the action remains optimal until the next event in the system. This result follows directly from the continuous-time Bellman equation (Puterman (2005), Chapter 11).

We formulate a 2-step value function, in order to separate transitions and actions and simplify the involved expressions. We define the dynamic programming value functions $U_{n}(.,$.$) and V_{n}(.,$.$) over n \geq 0$ steps, depending on the state of the system (the first variable is the state of the server pair, and the second variable is the number of customers in the queue). We express $V_{n+1}(.,$.$) in V_{n}(.,$.$) in the following$ way. First, the holding costs until the next jump are incurred by the cost function $c(.,$.$) , defined as$ $c(0, x)=\frac{c_{R}}{\lambda} x, c(i, x)=\frac{c_{R}}{\lambda}(x+1)$ for $i=1,2$ and $c(1 / 2, x)=\frac{c_{R}}{\lambda}(x+2)$, to account for the time spent in the system by a given customer. A cost of 1 is incurred per unsuccessful service. One of three events can happen: an arrival with probability $\frac{\lambda}{\lambda+\mu_{1}+\mu_{2}}$, a departure from server 1 with probability $\frac{\mu_{1}}{\lambda+\mu_{1}+\mu_{2}}$ or a departure from server 2 with probability $\frac{\mu_{2}}{\lambda+\mu_{1}+\mu_{2}}$. We assume without loss of generality that $\lambda+\mu_{1}+\mu_{2}=1$, such that the rate out of each state is equal to 1 . The rates are therefore considered 
as transition probabilities. We thus may write

$$
\begin{aligned}
V_{n+1}(0, x) & =\frac{c_{R}}{\lambda} x+\lambda U_{n}(0, x+1)+\left(\mu_{1}+\mu_{2}\right) U_{n}(0, x), \\
V_{n+1}(1, x) & =\frac{c_{R}}{\lambda}(x+1)+\lambda U_{n}(1, x+1)+\mu_{1}\left(U_{n}(0, x)+\alpha_{1}\right)+\mu_{2} U_{n}(1, x), \\
V_{n+1}(2, x) & =\frac{c_{R}}{\lambda}(x+1)+\lambda U_{n}(2, x+1)+\mu_{1} U_{n}(2, x)+\mu_{2}\left(U_{n}(0, x)+\alpha_{2}\right), \\
V_{n+1}(1 / 2, x) & =\frac{c_{R}}{\lambda}(x+2)+\lambda U_{n}(1 / 2, x+1)+\mu_{1}\left(U_{n}(2, x)+\alpha_{1}\right)+\mu_{2}\left(U_{n}(1, x)+\alpha_{2}\right),
\end{aligned}
$$

with

$$
\begin{gathered}
U_{n}(0, x)=\left\{\begin{array}{l}
V_{n}(0,0), \text { for } x=0 \\
\min \left(V_{n}(0,1), V_{n}(1,0), V_{n}(2,0)\right), \text { for } x=1 \\
\min \left(V_{n}(0, x), V_{n}(1, x-1), V_{n}(2, x-1), V_{n}(1 / 2, x-2)\right), \text { for } x>1,
\end{array}\right. \\
U_{n}(i, x)=\left\{\begin{array}{l}
V_{n}(i, 0), \text { for } x=0 \\
\min \left(V_{n}(i, x), V_{n}(1 / 2, x-1)\right), \text { for } x>0,
\end{array}\right.
\end{gathered}
$$

for $i \in\{1,2\}$, and

$$
U_{n}(1 / 2, x)=V_{n}(1 / 2, x)
$$

for $n, x \geq 0$. We choose for simplicity $U_{0}(.,)=$.0 and $V_{0}(.,)=$.0 . As explained in the next Section, the convergence of the value function is independent of choice of the initial condition.

For each $n>0$ and each state $(i, x)(i \in\{0,1,2,1 / 2\}$ and $x \geq 0)$, there is a minimizing action: serve two customers, serve one customer with server $i(i=1$ or $i=2)$ or do not serve any customer. For a fixed $n(n>0)$, the function

$\{0,1,2,1 / 2\} \times \mathbb{N} \rightarrow$ serve 2 customers, serve one customer with server $i$, do not serve,

is referred to as the customer routing policy at iteration $n$.

Remark. We assume in our modeling that a job cannot switch from one server to the other during service. In the opposite case, the optimal policy is known from George and Harrison (2001) since the system can be seen as an adjustable service rate queueing model. Server $i$ working alone is associated to a cost of $\alpha_{i} \mu_{i}$ and a service rate of $\mu_{i}(i=1,2)$ and both servers working together corresponds to a cost of $\alpha_{1} \mu_{1}+\alpha_{2} \mu_{2}$ and a service rate of $\mu_{1}+\mu_{2}$. The optimal policy would then be a two-thresholds policy. Under a first threshold the server with the lowest product $\alpha_{i} \mu_{i}(i=1,2)$ would be working alone, between this first threshold and a second one the server with the highest service rate would be working 
alone, and above this second threshold both servers should work.

\section{Optimal Routing}

One way of obtaining the infinite horizon average optimal actions is to use the value iteration technique introduced by Bellman (1957) and Howard (1960), by recursively evaluating $V_{n}$ using Equation (2), for $n \geq 0$. In Theorem 1, we prove by induction on the value function that the optimal policy is of threshold type. We prove that if some structural properties defining the threshold structure of the optimal policy are satisfied for $V_{n}$, then these properties are satisfied for $V_{n+1}$. They therefore hold for every $n$. As $n$ tends to infinity, the optimal policy converges to the unique average optimal policy, which is thus also of threshold type. This convergence result is ensured by Theorem 8.10.1 in Puterman (2005) since our problem satisfies the conditions of the theorem (countable state set, finite set of actions and uniformizable system). The proof of convergence to the average optimal policy is an important result in the MDP literature. It is based on showing that the iteration from $V_{n}$ to $V_{n+1}$ is a contraction mapping, as stated in Theorem 6.2.3 in Puterman (2005). This Theorem also proves that the optimal infinite horizon policy is independent of the choice of $V_{0}$. This is why one can simply choose $V_{0}(.,)=$.0 .

\subsection{The Result}

Theorem 1 The optimal routing policy is of threshold type. There exists a threshold $u(u>0)$ on the queue length $x$ such that:

- If $0 \leq x<u$, the optimal action is to maintain only one server idling.

- If $x \geq u$, it is optimal to have both servers busy.

The proof of Theorem 1 consists of three steps. They are first commented below. They are next proved in Section 3.2.

Step 1. The first step consists of proving that the number of servers which should be active follows a threshold policy. This threshold policy is characterized by the fact that if serving a customer is optimal in $x$, then serving a customer is also optimal in $x+1$. Sufficient conditions for this are

$$
V_{n}(0, x+1)-V_{n}(i, x) \geq 0 \Longrightarrow V_{n}(0, x+2)-V_{n}(i, x+1) \geq 0,
$$

and

$$
V_{n}(i, x+1)-V_{n}(1 / 2, x) \geq 0 \Longrightarrow V_{n}(i, x+2)-V_{n}(1 / 2, x+1) \geq 0,
$$


for $i=1,2$. These conditions are satisfied if

$$
V_{n}(0, x+2)+V_{n}(i, x)-V_{n}(0, x+1)-V_{n}(i, x+1) \geq 0,
$$

and

$$
V_{n}(i, x+2)+V_{n}(1 / 2, x)-V_{n}(i, x+1)-V_{n}(1 / 2, x+1) \geq 0,
$$

for $i=1,2$. The difference in the analysis here compared to that in Lin and Kumar (1984) or Koole (1995) is that we do not make a specific assumption on which server should be prioritized. The difficulty in the choice for server 1 or server 2 can be seen in the difference

$$
\begin{aligned}
V_{n+1}(1, x)-V_{n+1}(2, x) & =\lambda\left(U_{n}(1, x+1)-U_{n}(2, x+1)\right)+\mu_{1}\left(U_{n}(0, x)-U_{n}(2, x)\right) \\
& +\mu_{2}\left(U_{n}(1, x)-U_{n}(0, x)\right)+\mu_{1} \alpha_{1}-\mu_{2} \alpha_{2}
\end{aligned}
$$

for $x \geq 0$. With the last term, one could think of the routing control that routes customers in priority to the server that has the lowest unsuccessful service rate (minimum of $\mu_{i} \alpha_{i}$ for $i \in\{1,2\}$ ). However this simple rule does not propagate through value iterations because of the term $\mu_{1}\left(U_{n}(0, x)-U_{n}(2, x)\right)+$ $\mu_{2}\left(U_{n}(1, x)-U_{n}(0, x)\right)$ which can be either positive or negative. So, further assumptions are required to determine which server to prioritize.

Step 2. This step consists of proving, under an infinite horizon, that having the two servers idling at the same time cannot be optimal, as long as a waiting customer represents a strictly positive cost for the system. However, this statement cannot be proven by induction since both servers idling can be optimal under a finite horizon. For small $n$, not serving customers is often optimal: the costs of holding a customer in the queue over a short period can be cheaper than the costs of unsuccessful services. As an illustration, consider the problem with parameter values $\lambda=0.13, \mu_{1}=2, \mu_{2}=5, \alpha_{1}=0.1, \alpha_{2}=1$ and $c_{R}=0.005$. Using Equations (2) for $n=5$, we deduce that it is optimal to not serve any customer.

Since we are considering infinite horizon average performance, it would be tempting to first state that it is not optimal to have the two servers idling at the same time and next rewrite the definition of $U_{n}$ such that $U_{n}(0, x)=\min \left(V_{n}(1, x-1), V_{n}(2, x-1), V_{n}(1 / 2, x-2)\right)$ for $x>1$ and $U_{n}(0,1)=$ $\min \left(V_{n}(1,0), V_{n}(2,0)\right)$. Then less structural properties would need to be proven in the induction step from $V_{n}$ to $U_{n}$. However, this would force the system to make non-optimal decisions under finite horizon, and consequently not all structural properties required in Step 1 would to hold. For instance, as shown later in the induction from $V_{n}$ to $U_{n}$ in the proof for Relation (6), the proof of the last case $U_{n}(0, x+2)=$ $V_{n}(j, x+1)$ and $U_{n}(i, x)=V_{n}(i, x)$ would not go through. Instead of using a value iteration approach, 
we will prove this step of the proof by following a sample path approach.

Step 3. Assuming a threshold policy for the number of used servers (Step 1) and non-idling policies for both servers (Step 2), this last step consists of proving that whenever it is optimal to use only one server, the preference of which server it is cannot change.

\subsection{Proof of Theorem 1}

In what follows, we give the detailed proofs for the theorem three steps.

Step 1. We define the class of functions $\mathcal{F}$ from $\{0,1,2,1 / 2\} \times \mathbb{N}$ to $\mathbb{R}$ as follows: $f \in \mathcal{F}$ if for $x \geq 0$, we have

$$
\begin{aligned}
& f(i, x+1) \geq f(i, x), \text { for } i=0,1,2,1 / 2, \\
& f(1 / 2, x) \geq f(i, x) \geq f(0, x), \text { for } i=1,2, \\
& f(i, x+2)+f(1 / 2, x) \geq f(i, x+1)+f(1 / 2, x+1), \text { for } i=1,2, \\
& f(0, x+2)+f(i, x) \geq f(0, x+1)+f(i, x+1), \text { for } i=1,2, \\
& f(1 / 2, x+1)+f(i, x) \geq f(i, x+1)+f(1 / 2, x), \text { for } i=1,2, \\
& f(i, x+1)+f(0, x) \geq f(0, x+1)+f(i, x), \text { for } i=1,2 . \\
& f(i, x+1)+f(j, x) \geq f(0, x+1)+f(1 / 2, x), \text { for } i, j=1,2, i \neq j, \\
& f(0, x)+f(1 / 2, x) \geq f(1, x)+f(2, x) .
\end{aligned}
$$

If Relation (5) is true for $V_{n}$, then $V_{n}(i, x+2)-V_{n}(1 / 2, x+1) \geq V_{n}(i, x+1)-V_{n}(1 / 2, x)$, for $x \geq 0$. If $V_{n}(i, x+1)-V_{n}(1 / 2, x) \geq 0$, we thus deduce that $V_{n}(i, x+2)-V_{n}(1 / 2, x+1) \geq 0$, for $x \geq 0$. Consequently, if using server $j$ is optimal when $x$ customers are in the queue and server $i$ is busy $(i \neq j)$, then using server $j$ is also optimal when $x+1$ customers are in the queue and server $i$ is busy $(i \neq j)$. With Relation (6), the same observation holds for server $j$ when server $i$ is idle. Relations (5) and (6) for $V_{n}(n \geq 0)$ are then sufficient to prove that the optimal policy is of threshold type.

Observe that summing up Relation (5) and Relation (6) in which we replace $x$ by $x+1$, we obtain

$$
f(0, x+3)+f(1 / 2, x) \geq f(1 / 2, x+1)+f(0, x+2) .
$$

Note also that summing up Relation (5) and Relation (7) leads to the convexity in $x$ of $f(i, x)$; summing up Relation (5) and Relation (7) in which we replace $x$ by $x+1$ leads to the convexity in $x$ of $f(1 / 2, x)$; and summing up Relation (6) and Relation (8) leads to the convexity in $x$ of $f(0, x)$. 
Table 1: Summary of the proof

\begin{tabular}{c|cc}
\hline Relation to prove & \multicolumn{2}{|c}{ Follows from } \\
& A) $\left(V_{n}\right.$ to $\left.U_{n}\right)$ & B) $\left(U_{n}\right.$ to $\left.V_{n+1}\right)$ \\
\hline$(3)$ & $(3),(4)$ & $(3)$ \\
$(4)$ & $(3),(4)$ & $(4)$ \\
$(5)$ & $(5)$ & $(5),(6),(7)$ \\
$(6)$ & $(5),(6),(9)$ & $(6),(8)$ \\
$(7)$ & $(5),(7)$ & $(7),(8)$ \\
$(8)$ & $(3),(4),(5),(7),(8),(10)$ & $(8)$ \\
$(9)$ & $(7),(9)$ & $(8),(9)$ \\
$(10)$ & $(5),(7),(10)$ & $(10)$ \\
\hline
\end{tabular}

In Table 1 we summarize the required relations to prove each relation A) in the propagation from $V_{n}$ to $U_{n}$ (minimizing actions) and B) in the propagation from $U_{n}$ to $V_{n+1}$.

In what follows we prove by induction on $n$ that both $V_{n}$ and $U_{n}$ are in $\mathcal{F}$. For $x \geq 0, V_{0}(., x)=U_{0}(., x)=$ 0. Then $V_{0}, U_{0} \in \mathcal{F}$.

Induction from $V_{n}$ to $U_{n}$. Assume now that for a given $n \geq 0, V_{n} \in \mathcal{F}$, and let us prove that $U_{n} \in \mathcal{F}$. Relation (3): We have for $x \geq 0$,

$$
\begin{aligned}
& U_{n}(0, x) \leq V_{n}(0, x), \\
& U_{n}(0, x) \leq V_{n}(i, x-1), \text { for } x \geq 1, \\
& U_{n}(0, x) \leq V_{n}(1 / 2, x-2), \text { for } x \geq 2 .
\end{aligned}
$$

Case 1: $U_{n}(0, x+1)=V_{n}(0, x+1)$. Combining Inequality (12) with Relation (3) for $V_{n}$ proves Relation (3) for $U_{n}$. If $U_{n}(0, x+1)=V_{n}(i, x)$, then combining Inequality (13) with Relation (3) in the case $x \geq 1$ for $V_{n}$ proves Relation (3) for $U_{n}$. In the case $x=1$, combining Inequality (12) with Relation (4) for $V_{n}$ proves Relation (3) for $U_{n}$.

Case 2: $U_{n}(0, x+1)=V_{n}(1 / 2, x-1)$. Combining Inequality (14) in the case $x \geq 2$ with Relation (3) for $V_{n}$ proves Relation (3) for $U_{n}$. In the case $x=1$, combining Inequality (13) with Relation (4) for $V_{n}$ proves Relation (3) for $U_{n}$.

We have, for $x \geq 0$ and $i \in\{1,2\}$,

$$
\begin{aligned}
& U_{n}(i, x) \leq V_{n}(i, x), \\
& U_{n}(i, x) \leq V_{n}(1 / 2, x-1), \text { for } x \geq 1 .
\end{aligned}
$$

Case 1: $U_{n}(i, x+1)=V_{n}(i, x+1)$. Combining Inequality (15) with Relation (3) for $V_{n}$ proves Relation 
(3) for $U_{n}$.

Case 2: $U_{n}(i, x+1)=V_{n}(1 / 2, x)$. Combining Inequality (16) with Relation (3) in the case $x \geq 1$ for $V_{n}$ proves Relation (3) for $U_{n}$. In the case $x=1$, combining Inequality (15) with Relation (4) for $V_{n}$ proves Relation (3) for $U_{n}$.

Relation (4): We have, for $x \geq 0$ and $i \in\{1,2\}$,

$$
\begin{aligned}
& U_{n}(0, x) \leq V_{n}(0, x), \\
& U_{n}(0, x) \leq V_{n}(i, x-1), \text { for } x \geq 1 .
\end{aligned}
$$

Case 1: $U_{n}(i, x)=V_{n}(i, x)$. Combining Inequality (17) with Relation (4) for $V_{n}$ proves Relation (4) for $U_{n}$.

Case 2: $U_{n}(i, x)=V_{n}(1 / 2, x-1)$. Combining Inequality (18) with Relation (4) for $V_{n}$ proves Relation (4) for $U_{n}$.

We have, for $x \geq 0$ and $i \in\{1,2\}$,

$$
U_{n}(i, x) \leq V_{n}(1 / 2, x-1) \text { for } x \geq 1 .
$$

Also, $U_{n}(1 / 2, x)=V_{n}(1 / 2, x)$. Then combining Inequality (19) with Relation (3) for $V_{n}$ proves Relation (4) for $U_{n}$.

Relation (5): We have, for $x \geq 0$ and $i \in\{1,2\}$,

$$
\begin{aligned}
& U_{n}(i, x+1)+U_{n}(1 / 2, x+1) \leq V_{n}(i, x+1)+V_{n}(1 / 2, x+1), \\
& U_{n}(i, x+1)+U_{n}(1 / 2, x+1) \leq V_{n}(1 / 2, x)+V_{n}(1 / 2, x+1) .
\end{aligned}
$$

Also, $U_{n}(1 / 2, x)=V_{n}(1 / 2, x)$.

Case 1: $U_{n}(i, x+2)=V_{n}(i, x+2)$. Combining Inequality (20) with Relation (5) for $V_{n}$ proves Relation (5) for $U_{n}$.

Case 2: $U_{n}(i, x+2)=V_{n}(1 / 2, x+1)$. then Inequality (21) proves Relation (5) for $U_{n}$. 
Relation (6): We have, for $x \geq 0$ and $i \in\{1,2\}$,

$$
\begin{aligned}
& U_{n}(0, x+1)+U_{n}(i, x+1) \leq V_{n}(0, x+1)+V_{n}(i, x+1), \\
& U_{n}(0, x+1)+U_{n}(i, x+1) \leq V_{n}(0, x+1)+V_{n}(1 / 2, x), \\
& U_{n}(0, x+1)+U_{n}(i, x+1) \leq V_{n}(1 / 2, x-1)+V_{n}(1 / 2, x) \text { for } x \geq 1, \\
& U_{n}(0, x+1)+U_{n}(i, x+1) \leq V_{n}(i, x)+V_{n}(1 / 2, x), \\
& U_{n}(0, x+1)+U_{n}(i, x+1) \leq V_{n}(i, x)+V_{n}(i, x+1) .
\end{aligned}
$$

Case 1: $U_{n}(0, x+2)=V_{n}(0, x+2)$ and $U_{n}(i, x)=V_{n}(i, x)$. Combining Inequality (22) with Relation (6) for $V_{n}$ proves Relation (6) for $U_{n}$. For $x \geq 1$.

Case 2: $U_{n}(0, x+2)=V_{n}(0, x+2)$ and $U_{n}(i, x)=V_{n}(1 / 2, x-1)$. Combining Inequality (23) with Relation (11) for $V_{n}$ proves Relation (6) for $U_{n}$. For $x \geq 1$.

Case 3: $U_{n}(0, x+2)=V_{n}(1 / 2, x)$ and $U_{n}(i, x)=V_{n}(1 / 2, x-1)$. Inequality (24) proves Relation (6) for $U_{n}$.

Case 4: $U_{n}(0, x+2)=V_{n}(1 / 2, x)$ and $U_{n}(i, x)=V_{n}(i, x)$. Inequality (25) proves Relation (6) for $U_{n}$.

Case 5: $U_{n}(0, x+2)=V_{n}(i, x+1)$ and $U_{n}(i, x)=V_{n}(i, x)$. Inequality (26) proves Relation (6) for $U_{n}$.

Case 6: $U_{n}(0, x+2)=V_{n}(i, x+1)$ and $U_{n}(i, x)=V_{n}(1 / 2, x-1)$. Combining Inequality (25) with Relation (5) for $V_{n}$ proves Relation (6) for $U_{n}$.

Case 7: If $U_{n}(0, x+2)=V_{n}(j, x+1)$ and $U_{n}(i, x)=V_{n}(i, x)$. Combining Inequality (23) with Relation (9) for $V_{n}$ proves Relation (6) for $U_{n}$.

Relation (7): We have, for $x \geq 0$ and $i \in\{1,2\}$,

$$
\begin{aligned}
& U_{n}(1 / 2, x)+U_{n}(i, x+1) \leq V_{n}(1 / 2, x)+V_{n}(i, x+1), \\
& U_{n}(1 / 2, x)+U_{n}(i, x+1) \leq 2 V_{n}(1 / 2, x) .
\end{aligned}
$$

Also, $U_{n}(1 / 2, x)=V_{n}(1 / 2, x)$.

Case 1: $U_{n}(i, x)=V_{n}(i, x)$. Combining Inequality (27) with Relation (7) for $V_{n}$ proves Relation (7) for $U_{n}$.

Case 2: $U_{n}(i, x)=V_{n}(1 / 2, x-1)$. Combining Inequality (28) with the convexity in $x$ of $V_{n}(1 / 2, x)$ proves Relation (7) for $U_{n}$. 
Relation (8): We have for $x \geq 0$ and $i \in\{1,2\}$

$$
\begin{aligned}
& U_{n}(0, x+1)+U_{n}(i, x) \leq V_{n}(0, x+1)+V_{n}(i, x), \\
& U_{n}(0, x+1)+U_{n}(i, x) \leq 2 V_{n}(i, x), \\
& U_{n}(0, x+1)+U_{n}(i, x) \leq V_{n}(1, x)+V_{n}(2, x), \\
& U_{n}(0, x+1)+U_{n}(i, x) \leq V_{n}(1 / 2, x-1)+V_{n}(i, x), \text { for } x \geq 1, \\
& U_{n}(0, x+1)+U_{n}(i, x) \leq 2 V_{n}(1 / 2, x-1), \text { for } x \geq 1 .
\end{aligned}
$$

Case 1: $U_{n}(i, x+1)=V_{n}(i, x+1)$ and $U_{n}(0, x)=V_{n}(0, x)$. Combining Inequality (29) with Relation (8) for $V_{n}$ proves Relation (8) for $U_{n}$.

Case 2: $U_{n}(i, x+1)=V_{n}(1 / 2, x)$ and $U_{n}(0, x)=V_{n}(0, x)$. Combining Inequality (31) with Relation (10) for $V_{n}$ proves Relation (8) for $U_{n}$.

Case 3: $U_{n}(i, x+1)=V_{n}(i, x+1)$ and $U_{n}(0, x)=V_{n}(i, x)$. Combining Inequality (30) with Relation (3) for $V_{n}$ proves Relation (8) for $U_{n}$.

Case 4: $U_{n}(i, x+1)=V_{n}(1 / 2, x)$ and $U_{n}(0, x)=V_{n}(i, x)$. Combining Inequality (30) with Relation (4) for $V_{n}$ proves Relation (8) for $U_{n}$.

Case 5: $U_{n}(i, x+1)=V_{n}(i, x+1)$ and $U_{n}(0, x)=V_{n}(1 / 2, x-1)$. Combining Inequality (32) with Relation (5) for $V_{n}$ proves Relation (8) for $U_{n}$.

Case 6: $U_{n}(i, x+1)=V_{n}(1 / 2, x)$ and $U_{n}(0, x)=V_{n}(1 / 2, x-1)$. Inequality (33) with the convexity in $x$ of $V_{n}(1 / 2, x)$ proves Relation (8) for $U_{n}$.

Relation (9): We have, for $x \geq 0$ and $i, j=1,2(i \neq j)$,

$$
\begin{aligned}
& U_{n}(0, x+1)+U_{n}(1 / 2, x) \leq V_{n}(0, x+1)+V_{n}(1 / 2, x), \\
& U_{n}(0, x+1)+U_{n}(1 / 2, x) \leq V_{n}(j, x)+V_{n}(1 / 2, x), \\
& U_{n}(0, x+1)+U_{n}(1 / 2, x) \leq V_{n}(i, x)+V_{n}(1 / 2, x), \\
& U_{n}(0, x+1)+U_{n}(1 / 2, x) \leq V_{n}(1 / 2, x-1)+V_{n}(1 / 2, x), \text { for } x \geq 1 .
\end{aligned}
$$

Consider the case $i, j \in\{1,2\}$ and $i \neq j$.

Case 1: $U_{n}(i, x+1)=V_{n}(i, x+1)$ and $U_{n}(j, x)=V_{n}(j, x)$. Combining Inequality (34) with Relation (9) for $V_{n}$ proves Relation (9) for $U_{n}$.

Case 2: $U_{n}(i, x+1)=V_{n}(1 / 2, x)$ and $U_{n}(j, x)=V_{n}(j, x)$. Inequality (35) proves Relation (9) for $U_{n}$.

Case 3: $U_{n}(i, x+1)=V_{n}(i, x+1)$ and $U_{n}(j, x)=V_{n}(1 / 2, x-1)$. Combining Inequality (36) with Relation (7) for $V_{n}$ proves Relation (9) for $U_{n}$. 
Case 4: $U_{n}(i, x+1)=V_{n}(1 / 2, x)$ and $U_{n}(j, x)=V_{n}(1 / 2, x-1)$. Inequality (37) proves Relation (9) for $U_{n}$

Relation (10): We have, for $x \geq 0$,

$$
\begin{aligned}
& U_{n}(1, x)+U_{n}(2, x) \leq V_{n}(1, x)+V_{n}(2, x), \\
& U_{n}(1, x)+U_{n}(2, x) \leq V_{n}(1 / 2, x-1)+V_{n}(2, x), \text { for } x \geq 1, \\
& U_{n}(1, x)+U_{n}(2, x) \leq 2 V_{n}(1 / 2, x-1), \text { for } x \geq 1 .
\end{aligned}
$$

Also, $U_{n}(1 / 2, x)=V_{n}(1 / 2, x)$.

Case 1: $U_{n}(0, x)=V_{n}(0, x)$. Combining Inequality (38) with Relation (10) for $V_{n}$ proves Relation (10) for $U_{n}$.

Case 2: $U_{n}(0, x)=V_{n}(2, x-1)$. Combining Inequality (39) with Relation (7) for $V_{n}$ proves Relation (10) for $U_{n}$.

The case $U_{n}(0, x)=V_{n}(1, x-1)(x \geq 1)$ is identical.

Case 3: $U_{n}(0, x)=V_{n}(1 / 2, x-2)$. Combining Inequality (40) with the convexity in $x$ of $V_{n}(1 / 2, x)$ proves Relation (10) for $U_{n}$.

Induction from $U_{n}$ to $V_{n+1}$. Assume now that for a given $n \geq 0, U_{n} \in \mathcal{F}$. We next show that $V_{n+1} \in \mathcal{F}$.

Relations (3): We have, for $x \geq 0$,

$V_{n+1}(0, x+1)-V_{n+1}(0, x)=\lambda\left(U_{n}(0, x+2)-U_{n}(0, x+1)\right)+\left(\mu_{1}+\mu_{2}\right)\left(U_{n}(0, x+2)-U_{n}(0, x+1)\right)+\frac{c_{R}}{\lambda}$.

Since Relation (3) holds for $U_{n}$, the terms proportional to $\lambda$ and $\mu_{1}+\mu_{2}$ are positive. We thus conclude that Relation (3) is true for $V_{n}$.

We have, for $x \geq 0$ and $i \in\{1,2\}$,

$$
\begin{aligned}
V_{n+1}(i, x+1)-V_{n+1}(i, x) & =\lambda\left(U_{n}(i, x+2)-U_{n}(i, x+1)\right)+\mu_{i}\left(U_{n}(0, x+1)-U_{n}(0, x)\right) \\
& +\mu_{j}\left(U_{n}(i, x+1)-U_{n}(i, x)\right)+\frac{c_{R}}{\lambda}
\end{aligned}
$$

Since Relation (3) holds for $U_{n}$, the terms proportional to $\lambda$ and $\mu_{i}$ and $\mu_{j}$ are positive. We deduce that Relation (3) is true for $V_{n}$. 
We have, for $x \geq 0$ and $i \in\{1,2\}$,

$$
\begin{aligned}
V_{n+1}(1 / 2, x+1)-V_{n+1}(1 / 2, x) & =\lambda\left(U_{n}(1 / 2, x+2)-U_{n}(1 / 2, x+1)\right)+\mu_{1}\left(U_{n}(2, x+1)-U_{n}(2, x)\right) \\
& +\mu_{2}\left(U_{n}(1, x+1)-U_{n}(1, x)\right)+\frac{c_{R}}{\lambda} .
\end{aligned}
$$

Since Relation (3) holds for $U_{n}$, the terms proportional to $\lambda$ and $\mu_{i}$ and $\mu_{j}$ are positive. Thus Relation (3) is true for $V_{n}$ in this case.

Relation (4): We have, for $x \geq 0$ and $i \in\{1,2\}$,

$$
V_{n+1}(i, x)-V_{n+1}(0, x)=\lambda\left(U_{n}(i, x+1)-U_{n}(0, x+1)\right)+\mu_{i} \alpha_{i}+\mu_{j}\left(U_{n}(i, x)-U_{n}(0, x)\right)+\frac{c_{R}}{\lambda} .
$$

Since Relation (4) holds for $U_{n}$, the terms proportional to $\lambda$ and $\mu_{j}$ are positive. Moreover, $\mu_{i} \alpha_{i} \geq 0$. Thus Relation (4) is true for $V_{n}$ in this case.

We have, for $x \geq 0$ and $i \in\{1,2\}$,

$$
V_{n+1}(1 / 2, x)-V_{n+1}(i, x)=\lambda\left(U_{n}(1 / 2, x+1)-U_{n}(i, x+1)\right)+\mu_{i}\left(U_{n}(j, x)-U_{n}(0, x)\right)+\mu_{j} \alpha_{j}+\frac{c_{R}}{\lambda} .
$$

Since Relation (4) holds for $U_{n}$, the terms proportional to $\lambda$ and $\mu_{i}$ are positive. Moreover, $\mu_{j} \alpha_{j} \geq 0$. Thus, Relation (4) holds for $V_{n}$.

Relation (5): We have, for $x \geq 0$,

$$
\begin{aligned}
V_{n+1}(i, x+2) & +V_{n+1}(1 / 2, x)-V_{n+1}(i, x+1)-V_{n+1}(1 / 2, x+1) \\
=\lambda( & \left.U_{n}(i, x+3)+U_{n}(1 / 2, x+1)-U_{n}(i, x+2)-U_{n}(1 / 2, x+2)\right) \\
& +\mu_{i}\left(U_{n}(0, x+2)+U_{n}(j, x)-U_{n}(0, x+1)-U_{n}(j, x+1)\right) \\
& +\mu_{j}\left(U_{n}(i, x+2)+U_{n}(i, x)-2 U_{n}(i, x+1)\right),
\end{aligned}
$$

for $i, j=1,2$ and $i \neq j$. Since Relation (5) holds for $U_{n}$, the term proportional to $\lambda$ is positive. Since Relation (6) holds for $U_{n}$, the term proportional to $\mu_{i}$ is positive. Since $U_{n}(i, x)$ is convex in $x$, the term proportional to $\mu_{j}$ is positive. Thus, Relation (5) is true for $V_{n}$. 
Relation (6): We have, for $x \geq 0$,

$$
\begin{aligned}
V_{n+1}(0, x+2) & +V_{n+1}(i, x)-V_{n+1}(0, x+1)-V_{n+1}(i, x+1) \\
=\lambda( & \left.U_{n}(0, x+3)+U_{n}(i, x+1)-U_{n}(0, x+2)-U_{n}(i, x+2)\right) \\
+ & \mu_{i}\left(U_{n}(0, x+2)+U_{n}(0, x)-2 U_{n}(0, x+1)\right) \\
+ & \mu_{j}\left(U_{n}(0, x+2)+U_{n}(i, x)-U_{n}(0, x+1)-U_{n}(i, x+1)\right),
\end{aligned}
$$

for $i, j=1,2$ and $i \neq j$. Since Relation (6) holds for $U_{n}$, the terms proportional to $\lambda$ and $\mu_{j}$ are positive. Since $U_{n}(0, x)$ is convex in $x$, the term proportional to $\mu_{i}$ is positive. We therefore deduce that Relation (6) holds for $V_{n}$.

Relation (7): We have, for $x \geq 0$,

$$
\begin{gathered}
V_{n+1}(1 / 2, x+1)+V_{n+1}(i, x)-V_{n+1}(i, x+1)-V_{n+1}(1 / 2, x) \\
=\lambda\left(U_{n}(1 / 2, x+2)+U_{n}(i, x+1)-U_{n}(i, x+2)-U_{n}(1 / 2, x+1)\right) \\
\quad+\mu_{i}\left(U_{n}(j, x+1)+U_{n}(0, x)-U_{n}(0, x+1)-U_{n}(j, x)\right),
\end{gathered}
$$

for $i, j=1,2$ and $i \neq j$. Since Relation (7) holds for $U_{n}$, the term proportional to $\lambda$ is positive. Since Relation (8) holds for $U_{n}$, the term proportional to $\mu_{i}$ is positive. Therefore, Relation (7) is true for $V_{n}$. Relation (8): We have, for $x \geq 0$,

$$
\begin{array}{r}
V_{n+1}(i, x+1)+V_{n+1}(0, x)-V_{n+1}(0, x+1)-V_{n+1}(i, x) \\
=\lambda\left(U_{n}(i, x+2)+U_{n}(0, x+1)-U_{n}(0, x+2)-U_{n}(i, x+1)\right) \\
+\mu_{j}\left(U_{n}(i, x+1)+U_{n}(0, x)-U_{n}(0, x+1)-U_{n}(i, x)\right),
\end{array}
$$

for $i, j=1,2$ and $i \neq j$. Since Relation (8) holds for $U_{n}$, the terms proportional to $\lambda$ and $\mu_{j}$ are positive. Therefore, Relation (8) is true for $V_{n}$.

Relation (9): We have, for $x \geq 0$,

$$
\begin{aligned}
V_{n+1}(i, x+1) & +V_{n+1}(j, x)-V_{n+1}(0, x+1)-V_{n+1}(1 / 2, x) \\
=\lambda( & \left.U_{n}(i, x+2)+U_{n}(j, x+1)-U_{n}(0, x+2)-U_{n}(1 / 2, x+1)\right) \\
& +\mu_{j}\left(U_{n}(i, x+1)+U_{n}(0, x)-U_{n}(0, x+1)-U_{n}(i, x)\right),
\end{aligned}
$$


for $i, j=1,2$ and $i \neq j$. Since Relation (9) holds for $U_{n}$, the term proportional to $\lambda$ is positive. Since Relation (8) holds for $U_{n}$, the term proportional to $\mu_{j}$ is positive. Therefore, Relation (9) holds for $V_{n}$.

Relation (10): We have, for $x \geq 0$,

$$
\begin{aligned}
& V_{n+1}(0, x)+V_{n+1}(1 / 2, x)-V_{n+1}(1, x)-V_{n+1}(2, x) \\
& =\lambda\left(U_{n}(0, x+1)+U_{n}(1 / 2, x+1)-U_{n}(1, x+1)-U_{n}(2, x+1)\right) .
\end{aligned}
$$

Since Relation (10) holds for $U_{n}$, the term proportional to $\lambda$ is positive. Therefore, Relation (10) is true for $V_{n}$. This finishes the proof by induction of the first step of the proof.

Step 2. We prove this step using sample path arguments. Consider a scheduling policy $\pi$. Suppose that at time $t_{1}$, under policy $\pi$, the two servers are free while at least one customer is in the queue. This customer in the head of the line (oldest customer in the queue) is referred to as HoL. Assume that HoL has waited $w$ time units so far $(w \geq 0)$. For stability reasons, since the queue discipline is FCFS, there will be a later time instant, say $t_{2}$, where HoL will be scheduled in service to Server $i(i=1$ or $i=2$ ). This customer has waited $w+t_{2}-t_{1}$ time units before starting service and the probability of an unsuccessful service is $\alpha_{i}(i=1$ or $i=2)$. The next service starts at time $t_{3}$ with $t_{3} \geq t_{2}$ by one of the two servers.

Let us now construct the policy $\pi^{\prime}$ which follows exactly the same actions as $\pi$ except for HoL. Policy $\pi^{\prime}$ schedules HoL to Server $i$ at $t_{1}$ instead of $t_{2}$, but the next start of service is at time $t_{3}$ as under $\pi$. The difference between the two objective functions under $\pi$ and $\pi^{\prime}$ is then only related to the delaying or not of the start of service of HoL. Under $\pi^{\prime}$, this customer has waited $w$ time units before service and the probability of an unsuccessful service is still the same $\alpha_{i}$ as under $\pi$. Therefore under $\pi^{\prime}$, HoL has waited less than under $\pi$ while having the same probability of unsuccessful service. All remaining customers have the same waiting times and the same probabilities of unsuccessful services under both policies. Therefore $\pi^{\prime}$ outperforms $\pi$, which proves also that idling the two servers at the same time can never be optimal under the infinite horizon.

Step 3. In what follows, we prove that there cannot be any changes in the preference for one server if only one server should work. In other words, we prove that if server $i(i=1,2)$ is preferred when one job is in the system, then server $i$ should always work.

Consider a given $n$ for which the computation of the value function leads to the non-optimality of both servers idling. We therefore have $U_{n}(0,1)=\min \left(V_{n}(1,0), V_{n}(2,0)\right)$. If $U_{n}(0,1)=V_{n}(1,0)$ 
(preference for server 1), then from Relation (9) for $x=0, i=2$ and $j=1$, we obtain $V_{n}(2,1) \geq$ $V_{n}(1 / 2,0)$. This implies that if two jobs are in the system, it is either optimal to only use server 1 or to use both servers. Using server 2 only is then not optimal. From Relation (5) for $i=2$, we may write $V_{n}(2, x+2)-V_{n}(1 / 2, x+1) \geq V_{n}(2, x+1)-V_{n}(1 / 2, x)$ for $x \geq 0$. Thus for $x \geq 0$, we have $V_{n}(2, x+1)-V_{n}(1 / 2, x) \geq V_{n}(2,1)-V_{n}(1 / 2,0) \geq 0$. This proves that using server 2 only is never the optimal strategy. The same reasoning holds if $U_{n}(0,1)=V_{n}(2,0)$ (preference for server 2). This finishes Step 3 and the proof of the Theorem.

\subsection{Numerical Illustration}

In Figure 1, we illustrate the optimal policy as a function of the couple $(\lambda, x)$. We compute $V_{n}(.,$.$) using$ Equation (2) and stop the iterations until the following criterion is met

$$
\max _{i, x}\left\{V_{n+1}(i, x)-V_{n}(i, x)\right\}-\min _{i, x}\left\{V_{n+1}(i, x)-V_{n}(i, x)\right\}<\epsilon
$$

for $\epsilon=10^{-6}$. Figure 1(a) illustrates a situation where server 2 is prioritized since this server is at the same time the fastest and the most efficient. Figure 1(b) illustrates a situation where server 1 is prioritized although this server is the slowest. As expected we observe in both situations that the number of states, where both servers should work, increases with the arrival rate.

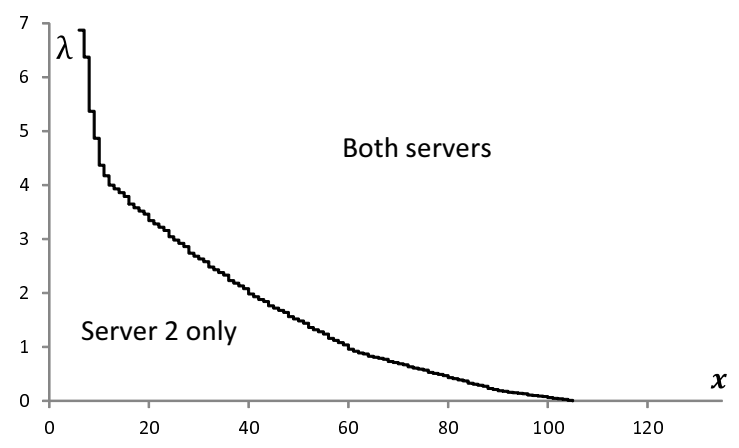

(a) $\alpha_{1}=80 \%, \alpha_{2}=10 \%$

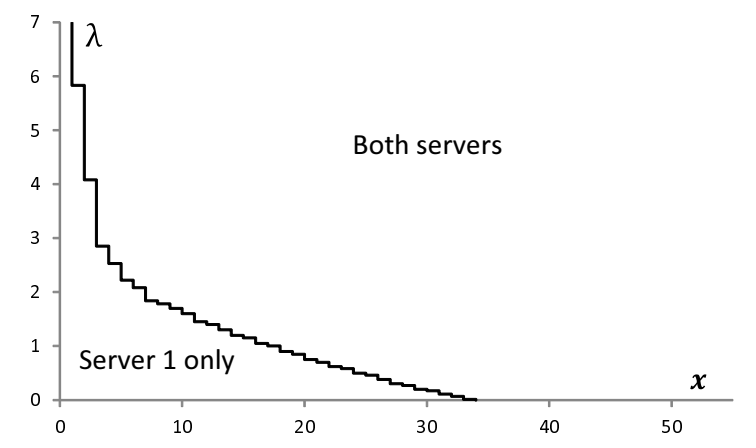

(b) $\alpha_{1}=10 \%, \alpha_{2}=80 \%$

Figure 1: Optimal Thresholds $\left(\mu_{1}=2, \mu_{2}=5, c_{R}=0.005 \lambda\right)$

Note that there is no known simple expression for the optimal threshold nor simple criterion for which server to prioritize. This can be seen in the iterative computation of the value function. If $\mu_{1}>\mu_{2}$, we obtain for $n=2$,

$$
V_{2}(2,0)-V_{2}(1,0)=\frac{c_{R}}{\lambda}\left(\mu_{1}-\mu_{2}\right)+(1+\lambda)\left(\alpha_{2} \mu_{2}-\alpha_{1} \mu_{1}\right)+\mu_{1} \mu_{2}\left(\alpha_{2}-\alpha_{1}\right) .
$$


This expression already gives the idea that if server 1 has at the same time the highest service rate $\left(\mu_{1}>\right.$ $\left.\mu_{2}\right)$, the lowest unsuccessful throughput $\left(\alpha_{1} \mu_{1}<\alpha_{2} \mu_{2}\right)$ and the lowest probability of an unsuccessful service $\left(\alpha_{1}<\alpha_{2}\right)$, then this server should be prioritized. This is however only a necessary condition. As $n$ increases, the expression of $V_{n}(2,0)-V_{n}(1,0)$ does not allow to determine a simple necessary and sufficient criterion for prioritizing server 1 . The same complexity holds when it comes to determining if the two servers should both work. It can be seen in the following expression, for $n=2$ and $\mu_{1}>\mu_{2}$,

$$
V_{2}(1 / 2,0)-V_{2}(1,1)=-\frac{c_{R}}{\lambda}\left(\mu_{2}+\lambda\right)+\alpha_{2} \mu_{2}\left(2-\mu_{2}\right)-\alpha_{1} \mu_{1}^{2}
$$

This expression indicates that the slower is the fastest server (server 1 here) or the more successful is the slowest server, the more likely the choice would be for having both servers working.

In Table 2 we summarize the effect of the routing choices on the performance measures.

Table 2: Effect of the routing decisions

\begin{tabular}{|c|c|c|c|}
\hline Queue size condition & Decision epoch & Decision & Decision Effect \\
\hline$x<u$ & $\begin{array}{c}2 \text { servers idle after } \\
\text { a service completion } \\
\text { or upon a task arrival and } x=0\end{array}$ & $\begin{array}{l}\text { Route a task } \\
\text { to the server which } \\
\text { minimizes } \alpha \mu\end{array}$ & $\begin{array}{l}\text { Reduce number of } \\
\text { unsatisfied customers } \\
\text { per time unit }\end{array}$ \\
\hline$x<u$ & $\begin{array}{c}2 \text { servers idle after } \\
\text { a service completion } \\
\text { or at a task arrival and } x=0\end{array}$ & $\begin{array}{l}\text { Route a task } \\
\text { to the faster server }\end{array}$ & $\begin{array}{l}\text { Reduce } \\
\text { service } \\
\text { time }\end{array}$ \\
\hline$x \geq u$ & $\begin{array}{l}1 \text { server idle after } \\
\text { a service completion } \\
\text { or at a task arrival }\end{array}$ & $\begin{array}{l}\text { Route a task } \\
\text { to the remaining } \\
\text { idle server }\end{array}$ & $\begin{array}{l}\text { Reduce } \\
\text { waiting } \\
\text { time }\end{array}$ \\
\hline
\end{tabular}

\section{Conclusion}

The optimal job routing for the two heterogeneous server problem with quality of resolution follows a threshold policy on the queue size, defined by the threshold $u(u>0)$. After a service completion, if two servers are idling and $x$ jobs are in the queue $(0<x<u)$ or after a job arrival at an empty queue and the two servers are idling, it is optimal to route a job to only one of the two servers. The server to be prioritized depends on the relative importance given to the expected time spent in the system in comparison with the unsatisfied rate. After a service completion or an arrival, if $x \geq u$, it is optimal to have the two servers busy.

The optimal routing is intuitive. When the queue size is small, the expected waiting time is small for arriving jobs and the main concern of the manager is then to minimize the unsatisfied customers rate or the service times. Above the threshold on the queue size, the major problem for the manager becomes the waiting time and the two servers are then both requested to reduce it. 
An interesting but challenging topic for future research is to extend the results to the multi-server case. It would be also interesting to consider the control problem in a more general context with customer abandonments.

\section{References}

Armony, M. (2005). Dynamic routing in large-scale service systems with heterogeneous servers. Queueing Systems, 51(3):287-329.

Armony, M. and Ward, A. (2010). Fair dynamic routing in large-scale heterogeneous-server systems. Operations Research, 58(3):624-637.

Atar, R. (2008). Central limit theorem for a many-server queue with random service rates. The Annals of Applied Probability, 18(4):1548-1568.

Atar, R. and Shwartz, A. (2008). Efficient routing in heavy traffic under partial sampling of service times. Mathematics of Operations Research, 33(4):899-909.

Bellman, R. (1957). Dynamic Programming. Princeton University Press, Princeton.

Cabral, F. (2005). The slow server problem for uninformed customers. Queueing systems, 50(4):353-370.

de Véricourt, F. and Zhou, Y. (2005). Managing response time in a call-routing problem with service failure. Operations Research, 53(6):968-981.

de Véricourt, F. and Zhou, Y. (2006). On the incomplete results for the heterogeneous server problem. Queueing Systems, 52(3):189-191.

Efrosinin, D. (2013). Queueing model of a hybrid channel with faster link subject to partial and complete failures. Annals of Operations Research, 202(1):75-102.

George, J. M. and Harrison, J. M. (2001). Dynamic control of a queue with adjustable service rate. Operations Research, 49(5):720-731.

Hall, J. and Porteus, E. (2000). Customer service competition in capacitated systems. Manufacturing $\mathcal{G}$ Service Operations Management, 2(2):144-165.

Howard, R. (1960). Dynamic Programming and Markov Processes. Massachusetts Institute of Technology Press, Cambridge.

Jouini, O., Dallery, Y., and Nait-Abdallah, R. (2008). Analysis of the impact of team-based organizations in call centers management. Management Science, 54(2):400-414. 
Koole, G. (1995). A simple proof of the optimality of a threshold policy in a two-server queueing system. Systems \& Control Letters, 26(5):301-303.

Krishnamoorthi, B. (1963). On poisson queue with two heterogeneous servers. Operations Research, 11(3):321-330.

Larsen, R. and Agrawala, A. (1983). Control of a heterogeneous two-server exponential queueing system. IEEE Transactions on Software Engineering, (4):522-526.

Lin, W. and Kumar, P. (1984). Optimal control of a queueing system with two heterogeneous servers. IEEE Transactions on Automatic Control, 29(8):696-703.

Luh, H. P. and Viniotis, I. (2002). Threshold control policies for heterogeneous server systems. Mathematical Methods of Operations Research, 55(1):121-142.

Mehrotra, V., Ross, K., Ryder, G., and Zhou, Y. (2012). Routing to manage resolution and waiting time in call centers with heterogeneous servers. Manufacturing $\& 3$ Service Operations Management, 14(1):66-81.

Özkan, E. and Kharoufeh, J. (2014). Optimal control of a two-server queueing system with failures. Probability in the Engineering and Informational Sciences, 28(04):489-527.

Özkan, E. and Kharoufeh, J. (2015). Incompleteness of results for the slow-server problem with an unreliable fast server. Annals of Operations Research, 226(1):741-745.

Puterman, M. (2005). Markov Decision Processes. John Wiley and Sons, New Jersey.

Rubinovitch, M. (1985). The slow server problem. Journal of Applied Probability, 22(01):205-213.

Rykov, V. (2001). Monotone control of queueing systems with heterogeneous servers. Queueing Systems, 37(4):391-403.

Rykov, V. V. and Efrosinin, D. V. (2009). On the slow server problem. Automation and Remote Control, 70(12):2013-2023.

Viniotis, I. and Ephremides, A. (1988). Extension of the optimality of the threshold policy in heterogeneous multiserver queueing systems. IEEE Transactions on Automatic Control, 33(1):104-109.

Walrand, J. (1984). A note on "Optimal control of a queuing system with two heterogeneous servers". Systems \& Control Letters, 4(3):131-134.

Weber, R. (1993). On a conjecture about assigning jobs to processors of differing speeds. IEEE transactions on Automatic Control, 38(1):166-170. 
Yankovic, N. and Green, L. (2011). Good nursing levels: A queuing approach. Operations Research, $59(4): 942-955$.

Zhan, D. and Ward, A. (2014). Threshold routing to trade off waiting and call resolution in call centers. Manufacturing \&3 Service Operations Management, 16(2):220-237. 